\title{
Experimental Results and Proposed Electromechanical Model for Vibrational Communication in Mining and Oil-Gas Drill Strings
}

\author{
Md Shahriar Islam \\ Department of Mechanical Engineering \\ University of Saskatchewan \\ Saskatoon, SK, Canada \\ shahriar.islam@usask.ca \\ Nathan Peter \\ Development Engineering \& Manufacturing, Mining and \\ Minerals \\ Saskatchewan Research Council, Saskatoon, SK, Canada \\ Nathan.Peter@src.sk.ca
}

\author{
Travis Wiens \\ Department of Mechanical Engineering \\ University of Saskatchewan \\ Saskatoon, SK, Canada \\ t.wiens@usask.ca
}

\begin{abstract}
In borehole mining and oil and gas drilling, the drilling process is invisible from the surface, which is why a two-way communication method between the drill head and the surface is a must. The popular industrial instrumentation technique, widely known as measurement while drilling (MWD), has enabled industries to save costly employee hours and gain more control over the drill bit direction. There are four major methods used throughout history, where the mud pulse telemetry technique has become the state of the art because of its simplicity and convenience. However, like other methods, it has a major drawback of having a slow data transmission rate. Moreover, other methods have different issues which include large attenuation of the data that is transferred through drill pipes. Acoustic telemetry is the most recent method of transferring data through a solid medium, a technique using continuous wave propagation of forces in the wall of the pipe. A proper use of this new method is expected to become popular in the mining and oil-gas industries because of its faster data transmission rate and ability to ignore most attenuating parameters. This method is still in its optimization stage, and the use of a convenient tool to match the transducer with the parameters of the drill pipe and surroundings is still a challenge to overcome. This paper will propose a simulation model to simulate the transmission of data through drill pipes, and will show experimental results of such arrangement justifying the opportunities ahead in this field.
\end{abstract}

Keywords-acoustics; vibration; drill pipe; transmission line; communication; frequency response;

\section{INTRODUCTION}

Experts involved in surveying or extracting fossil fuel and minerals face a great challenge of drilling without seeing, which is why their usual methods include measuring real time data while drilling. This is commonly known as measurement while drilling (MWD) or logging while drilling (LWD). In addition, these operations involve large assemblies of equipment and inconveniences due to invisibility, complex well patterns, long operation times, high costs etc. The initial goal is to determine ore location, drilling orientation, properties of rock, dimensions of cavity and other parameters using sensor data assembled in the borehole assembly (BHA). This data is then transmitted to the surface from the wellbore (or the hole). Using basic trigonometry, a three-dimensional plot of the path of the well can be produced thus enabling an operator to measure the trajectory of the hole as it is drilled in real time. Therefore, it becomes easy to follow the preplanned direction of the drilled hole. Also, measurements to determine properties of the rock is done by taking natural gamma ray emissions from the rock [1]. Other information such as density, porosity, rock fluid pressures are taken by sensors based on sound or electricity [2]. All of this information helps to broadly determine what type of rock formation is being drilled, which in turn helps confirm the real-time location of the wellbore by comparing to seismic data. MWD downhole tools also allow the wellbore to be directed in a chosen direction, therefore known as directional drilling.

Historically there are four communication methods used, namely cable operated communication, mud pulse telemetry, vibration/pressure wave transmission and electromagnetic communication [3]. Among these, the most popular is mud pulse telemetry (MPT) which is a binary coding system and is the first of its kind to be used in the industry. It uses drilling fluid to transmit the pressure pulse created by a poppet type valve. The valve changes the drilling fluid's pressure thus the pressure wave propagates through the drilling fluid. At the surface the pressure differences are recorded and analyzed. This method, however, has its own issues such as 
non-homogeneity of the mud fluid and is only operational when the drilling fluid is running. Most importantly, due to signal attenuation in the fluid, its data transmission rate is slow on the scale of 1-10 bits per second. As a result, exploring other methods such as communication via structural vibration have become necessary [3].

Acoustic telemetry includes the use of piezo transducers to produce compression, torsion, or shear waves that propagate through the drill strings to the other end where they can be picked up using another piezo transducer or another type of sensor. The medium of pressure wave transmission is the solid steel pipe rather than viscous liquid as in mud pulse telemetry. The information picked up by different sensors is converted into digital data and is recorded into the memory and broadcast by the transducers as vibration or acoustic wave through the drill string. The transducer generates this vibration within the steel drill string by means of a high frequency ferroelectric ceramic piezoelectric material with the combination of two different masses [4]. The captured signal is transferred to a computer where a program decodes the digitized sensor signal and extracts the downhole information; this method can give faster data rates up to 100 bits per second [5]. The vibrational method and theories have been known from the beginning of the MWD, but because of the lack of an efficient transducer, the method did not flourish. Currently, because of advancements in piezoelectric ceramic technology, the method has resurfaced and is beginning to gain commercial popularity [6-9].

Acoustic telemetry uses the solid medium of the drill string itself and not the mud fluid which is why it has different attenuation. For typical oil and gas applications, the attenuation for acoustics systems varies from $2-10 \mathrm{~dB} / 1000$ feet and up to $30 \mathrm{~dB} / 1000$ feet if the pipe is badly worn [10-12]. Along with a number of patents, the work published in academic journals are found to focus on wave transmission of different types through mass jointed pipe strings [13-14]. Drumheller has done a considerable amount of work which included investigations of the basic theory of wave propagation, the hardware of communication tools, and collecting and explaining a history of industrial experimental results on attenuation. [15]. Carcione introduced a time-domain algorithm for the propagation of onedimensional axial, torsional, and flexural stress waves, which also includes transducer sources and sensors [16]. Based on this work some other works were modified and justified by experimental verification. Among the modifications include avoiding lateral vibration at the neutral point and a method to avoid them by a new approach of MPT and summarization of many more applications, such as wave loadings on offshore platforms, classical problems in wave propagation, and extensions to modern kinematic wave theory [17].

Most of the research work regarding drill bit communication focuses on communication through drill strings in the oil-gas industry but none address borehole mining, which has a number of important differences. In borehole mining, drill stems tend to be shorter, and larger in diameter and the whole pipeline can be within hundreds of meters, consisting of hundreds of pipes. Moreover, the impedance mismatch at each

Saskatchewan Research Council. (sponsor) pipe joint is much less, because the pipe joints are screwed to each other with no extension in outside diameter as seen in the pipe joints of the oil-gas drill pipe. So, the associated reflection and resonances at the pipe joints are less.

In this paper a universal simulation model will be proposed that can account for any types of drill string along with the transmitter and receiver for acoustic wave propagation. The simulation model will be verified by experimental data, which will justify the model. A simulation model will be a convenient approach to understand the characteristics of the pipelines for different applications, which include the attenuation and frequency shifting of the system. Moreover, this will lead to the optimization and modification of the transducer and sensors to be used with any specific application. If the frequency response of the system is known then a particular communication scheme can be chosen beforehand which can employ different frequency contents for a faster data transmission. Future research works will be to optimize the transducer based on the application and convenience in the communication scheme and also to choose a particular communication scheme.

\section{EXPERIMENTAL MODELING}

An experimental apparatus was assembled for data transmission through drill strings as shown in Fig. 1. This experimental model includes Tonpilz-type piezoelectric transducers as both the transmitter and receiver. These transducers were designed for sonar applications. It is possible to use an accelerometer sensor at the receiving end, but in this experiment an identical transducer was used as the receiver. This configuration allows for two-way communication to occur if needed.

\section{A. Frequecny Response of the Transducer}

First, the experiment is performed attaching the transducers face to face with bolts. This experiment gives the frequency response of the two transducers shown in Fig. 2.

All control and data measurements are done by a National Instruments data acquisition system (NI-DAQ). A maximum length sequence (MLS) is a digital pseudorandom binary sequence that has a perfectly white, flat spectrum. This signal is used as an input signal into the driver transducer through an amplifier. The MLS is generated using linear feedback shift registers (LFSR) using Matlab® code created by Wiens [18] and

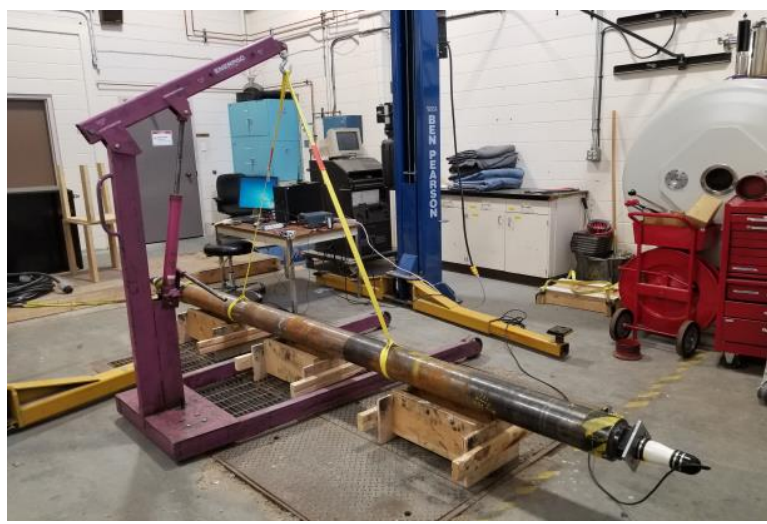

Figure 1. Arrangement for three pipes connected with transducers. 


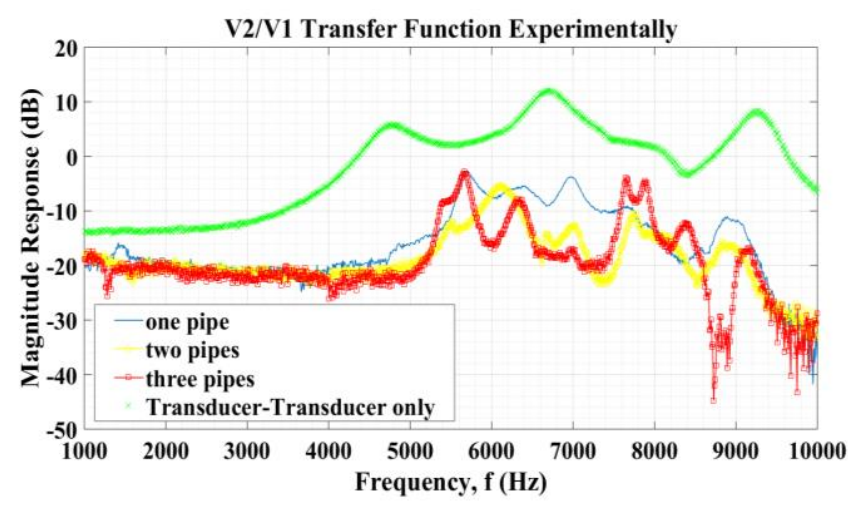

Figure 2. Frequecny Response of the transducers only connected face to face and transducers connected with one, two and three drill strings.

LSFR feedback values from Koopman [19]. The output is measured from the receiver transducer through a voltage divider which is recorded as voltage $\mathrm{v}_{2}$. The input voltage $\mathrm{v}_{1}$ is taken from the amplifier output through the voltage divider. The voltage divider is used here so that NI-DAQ device stays within the rated range of $\pm 10 \mathrm{~V}$, while the amplifier outputs $\mathrm{v}_{1}=100 \mathrm{~V}$. The MLS used has a sampling frequency of $20 \mathrm{kHz}$.

The measured data was then used to obtain transfer function

$$
\mathrm{ETFE}=\frac{\mathrm{v}_{2}}{\mathrm{~V}_{1}}
$$

where $V_{1}$ and $V_{2}$ are the fast Fourier transform (FFT) of the measured voltage $\left(\mathrm{v}_{1}\right.$ and $\left.\mathrm{v}_{2}\right)$ signals. This experimental method works well for pressure measurements of fluid transmission line [20] and is believed to be a good experimental approach for measuring vibration along any transmission line.

\section{B. One, Two and Three Drill Strings}

The transducers are connected with one drill string through the end caps. The drill string used for this experiment is collected from a mine site (in this application they are called jet rods). The transducers are then connected with two and three drill segment strings through end caps as shown in Fig. 1.

Fig. 2 shows the frequency response for different pipe arrangements with the transducers. It is clear from the figure that the frequency response range of the transducers is $4000 \mathrm{~Hz}$ to $10000 \mathrm{~Hz}$. For ease of understanding, the peak natural frequencies are extracted and Fig. 6 is formed to show that with the increase of pipes the natural frequencies of certain modes are reducing and more frequency contents become visible in the range.

\section{PROPOSED Simulation MODEL}

A model shown in Fig. 3 is proposed in this section for simulation that can represent the experimental apparatus previously shown. A successful model that can account for a certain number of drill strings and comply with the experimental results must be able to account for a large number of drill strings connected together. The model includes a driver/transmitter transducer, then a block for a number of drill strings and a receiver transducer to act as a sensor.
To obtain an effective and more practical simulation model, the linearized one-dimensional piezoelectric ceramic equations found in Sherman [21] are used. Also, the transducer is considered as a simple mass-spring-damper model where piezoelectric stack materials are placed between the head mass and tail mass. The pipeline of a finite number of drill strings can be modeled using a number of transmission line models [22] connected together. This accounts for the input and output forces and displacements in terms of transfer functions that can calculate the system more in a computationally inexpensive way.

In Fig. 3, $v_{1}$ is the input forcing voltage at the driver transducer, $\mathrm{u}_{1}$ is the displacement at the driver transducer, $\mathrm{F}_{1}$ is the output mechanical force of driver transducer and also the input of the pipeline. $F_{2}$ is the input force at the outlet of transmission/pipeline and also the output from the receiver transducer. Similarly, $\mathrm{u}_{1}$ and $\mathrm{u}_{2}$ are the displacements at the inlet and outlet of the transmission line. $\mathrm{v}_{2}$ is the output voltage from the receiver transducer.

\section{A. Piezoelectirc Transducer}

A piezoelectric transducer is an electromechanical device that converts mechanical energy into electrical energy or vice versa. A lot of previous research has been performed on the design of an effective transducer, among them is a $50 \mathrm{kHz}$ Tonpilz type transducer developed by Baylis [23]. A detailed discussion on the basic design analysis of this type of transducer is made in the book by Sherman and Butler [21]. This particular transducer is comprised of a combination of two masses bolted together with piezoelectric ceramic placed in between.

The basic governing equations of piezoelectric ceramics are the combination of mechanical and electrical characteristics which are dependent on each other. The constitutive relations are usually called piezoelectric equations that apply the theory of piezoelectricity. The equation, describe the interaction effects of stress, strain, electric displacement, and the electric field. In the case where the strain and electric fields are approximately uniform, the mass of the piezoelectric material can be assumed negligible, and the amount of free charge is small, the behavior of the transducer can be modelled by the simplified constitutive equations given in [21]

$$
\begin{aligned}
& S=s^{E} T+d^{t} E \\
& D=d T+\varepsilon{ }^{T} E
\end{aligned}
$$

where $\mathrm{T}$ is the stress, $\mathrm{S}$ is the strain, $\mathrm{E}$ is the electric field, and $\mathrm{D}$ is the electric displacement. All of these variables are functions of position and time, where $\mathrm{T}$ and $\mathrm{S}$ are both symmetric second rank tensors. In these equations, $\mathrm{S}$ and $\mathrm{T}$ are $6 \times 1$ column matrices, $E$ and $D$ are $3 \times 1$ column matrices, $s^{\mathrm{E}}$ is a

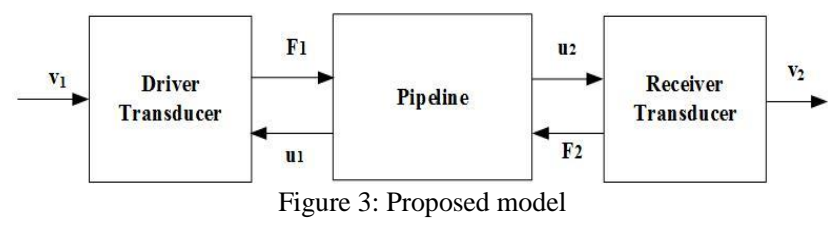


$6 \times 6$ matrix of elastic compliance coefficients, $d$ is a $6 \times 3$ matrix of piezoelectric coefficients ( $\mathrm{d}^{\mathrm{t}}$ is the transpose of $\mathrm{d}$ ), and $\varepsilon^{\mathrm{T}}$ is a $3 \times 3$ matrix of permittivity coefficients [24].

\section{B. Pipeline}

To model the drill string, a different approach is taken in this research by using a transmission line model which is often used in the hydraulic circuit design for fluid flow through a pipe. The transmission line model was initially developed by Krus [22] and is based on the assumption of a four pole equation and approximating the response with a number of transfer functions, as shown in Fig. 4. This model reduces to a compact transfer function model which only calculates the sates at inlet and outlet thus reducing the computational effort. There is other research done on the modification of this transmission line model in order to gain better accuracy and apply to other transmission line problems [25-27]. However, all of these are used in the application of fluid mechanics. For this research, pressure and flow are replaced with analogous force and velocity.

From analogy, in this model the pressure $\mathrm{P}$ and flow rate, $\mathrm{Q}$, can be replaced with force, $\mathrm{F}$, and velocity $\mathrm{u}$. They are related by the following equation [22]

$$
\begin{aligned}
& \mathrm{F}_{1}=\mathrm{C}_{1}+\mathrm{Z}_{\mathrm{c}} \mathrm{u}_{1} \\
& \mathrm{~F}_{2}=\mathrm{C}_{2}+\mathrm{Z}_{\mathrm{c}} \mathrm{u}_{2}
\end{aligned}
$$

where, $Z_{c}=A c \rho \quad$ is the characteristic impedance of the drill string, $C$ is the local speed of sound in the transmission line, $A$ is the cross-sectional area of the pipe, and $\rho$ is the material density of the drill string.

The transfer functions in Fig. 4 are as follows. They are calculated in the laplace domain, $s$.

$$
\begin{gathered}
\mathrm{H}_{1}(\mathrm{~s})=\frac{\mathrm{R}}{\mathrm{kTs}+1} \\
\mathrm{H}_{2}(\mathrm{~s})=\mathrm{Z}_{\mathrm{c}} \\
\mathrm{G}_{\mathrm{f}}(\mathrm{s})=\frac{\mathrm{kTe}^{\frac{-\mathrm{RA}}{2 \rho \mathrm{c}}} \mathrm{s}+1}{\mathrm{kTs}+1}
\end{gathered}
$$

where, $\mathrm{k}$ is an empirical factor. It was determined in [22] that $k=1.25$ is an acceptable value. The wave propagation time, $T=\frac{L}{c}$, is related to length of the pipe, L, and local speed of sound, c. The distributed line resistance, $R=\frac{\mu \pi \mathrm{d}}{h} L$, assuming ideal laminar flow. This model neglects unsteady friction, and assumes a distributed resistance across the transmission line.

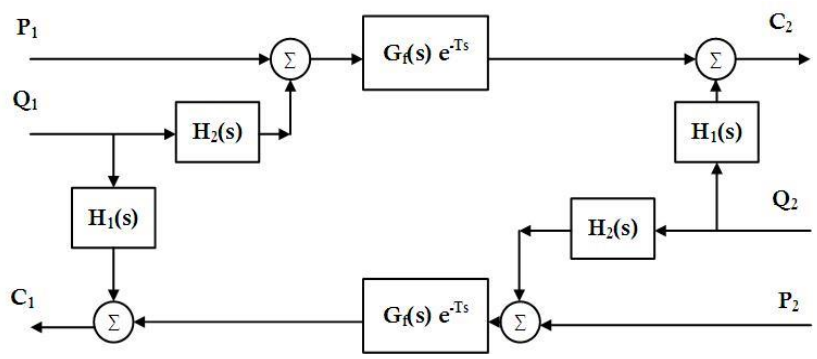

Figure 4. Block diagram of a fluid transmission line model [22].
The $e^{-T s}$ term with the $(s)$ transfer function is a time delay of time, $T$, simulating wave propagation.

\section{RESUlts OF SiMULATION MODEL AND COMPARISON}

The simulation model for the two transducers connected face to face show similar frequency response to that of experimental results except in the $1^{\text {st }}$ mode shown in Fig. 5, which is expected given the assumptions in the model. The assumptions are taken as the model consists of two mass spring damper system where the mass of the piezo ceramic is ignored and it is considered as a spring damper connection in between two masses [21]. Also, the actual transducer has masses bolted together at an unknown preload and the whole assembly is covered with neoprene type rubber material. So, these factors played an important role of moving the first mode of the experiment from the simulation model value.

Fig. 5 shows the frequency response of different pipe arrangements for the simulation model. The frequency response of different pipes have similar trend to that observed in the experimental result shown in Fig. 2.

Fig. 6 compares the resonant frequencies between the experimental apparatus and simulation model. The simulation model has a different range than the experimental model as shown in Fig. 5, which is why a different range is seen in the

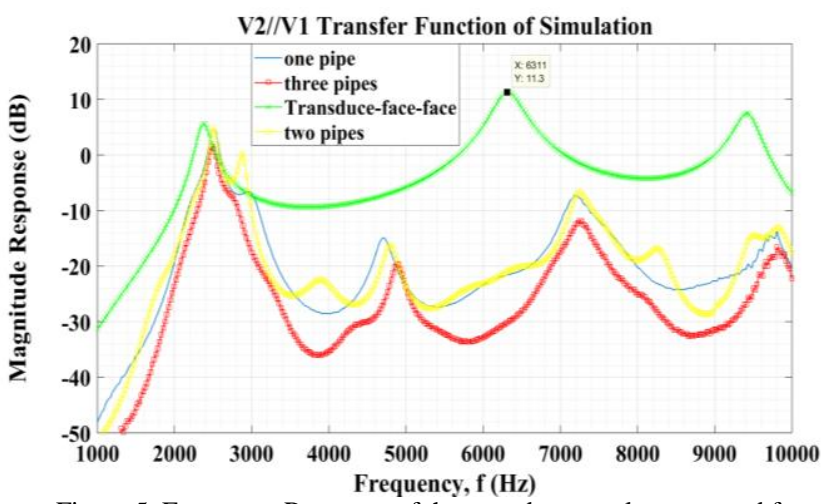

Figure 5. Frequecny Response of the transducers only connected face to face and transducers connected with one, two and three drill strings.

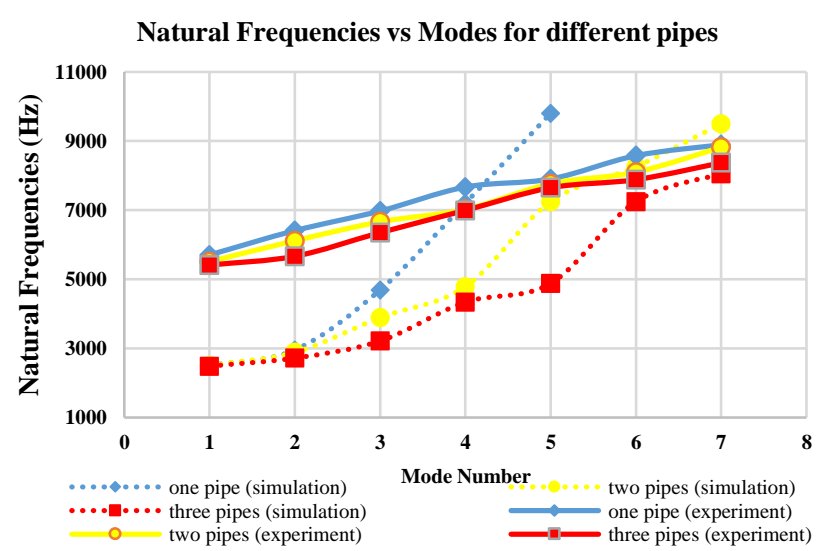

Figure 6. Comparison of Natural Frequencies of different Modes of different pipe arrangements of simulation model and experimental model. 
simulation model for the frequency response of different pipes than the experimental results. Also because the experimental apparatus is a drill string that has a construction of concentric inner and outer pipes supported by spacers of different materials, whereas in the simulation model a cylindrical type of pipe is considered with a similar mass matched with the experimental apparatus. However, a similar trend of decreasing natural frequencies of different modes with the increase of number of pipes is observed in both experimental and simulation model results. This is expected because the natural frequencies of a system change disproportionally with the square root of its mass.

\section{CONCLUSION}

The comparison of results between the simulation model and the experiment indicates that the simulation model is able to explain the wave propagation in long drill strings. Thus this can help with developing a communication scheme for data communication in drill string using methods that employ wide range of frequency content in a single transmission.

\section{ACKNOWLEDGMENT}

The authors would like to acknowledge the help of Mr. Douglas V. Bitner of University of Saskatchewan for his help with this research. The authors also acknowledge the support given by the Saskatchewan Research Council, SK, Canada for the experimental assembly and equipment, as well as financial support of this work.

\section{REFERENCES}

[1] J. J. Arps and J. L. Arps, "The subsurface telemetry problem - a practical solution," Journal of Petroleum Technology. Society of Petroleum Engineers, Vol. 16, Issue 05, May 1964. doi.org/10.2118/710-PA

[2] I. Dowell, A. Mills and M. Lora, "Chapter 15 - Drilling-Data Acquisition," In R. F. Mitchell. Petroleum Engineering Handbook. II Drilling Engineering. Society of Petroleum Engineers. pp. 647-685, 2006. ISBN 978-1-55563-114-7.

[3] N. G. Franconi, A. P. Bunger, E. Sejdic and M. H. Mickle, "Wireless communication in oil and gas wells," Energy Technology, vol. 2, no. 12, pp. 996-1005, 2014.

[4] M. S. Islam, A. T. Dolovich, N. Peter and T. Wiens, "Acoustic drill pipe communications for mining," Proceeding of the Maintenance, Engineering and Reliability/Mine Operators Conference, Canadian Institute of Mining, Metallurgy and Petroleum (CIM), 2017. http://store.cim.org/en/acoustic-drill-pipe-communications-for-mining

[5] D. S. Drumheller, S. S. Kuszmaul, "Acoustic Telemetry," Geothermal Research Department Sandia National Laboratories, Albuquerque, 2003. NM 87185-1 033

[6] F. Dias, F. Marcancola, D. Wakabayashi and R. Piazza, "First real-time drill-stem test in deepwater using fully acoustic telemetry monitoring and control of the well," Abu Dhabi International Petroleum Exhibition and Conference. Society of Petroleum Engineers, 2014.

[7] J. R. Haddad, A. Salguero, C. Jaimes, "Application of telemetry technology in high-pressure wells to improve data accuracy in drill stem tests," SPE Oil and Gas India Conference and Exhibition. Society of Petroleum Engineers, 2017.
[8] E. Kreuzer, L. Krumm and M. A. Pick, "Investigation of the influence of screw connections in drill-strings on the propagation of torsional waves with respect to advanced stick-slip controllers," 33rd International Conference on Ocean, Offshore and Arctic Engineering. American Society of Mechanical Engineers, pp. V005T11A022-V005T11A022, 2014.

[9] S. Fuchao, P. Xiaohan, Z. Lichen, W. Guoqing and J. Deli, "Research and validation of pipe acoustic transmission mechanism," International Journal of Multimedia and Ubiquitous Engineering, vol. 9, no. 11, pp. 161-170, 2014.

[10] W. Cox and P. Chaney, 1981, “Telemetry system," US Patent 4,293,936. 1981.

[11] D. S. Drumheller, "Acoustic data transmission through a drillstring," US Patent 5,128,901, 1992.

[12] J. Orban and R. Squire, "Sonic vibration telemetering system," US Patent $5,373,481,1994$.

[13] T. G. Barnes and B. R. Kirkwood, "Passbands for acoustic transmission in an idealized drill string," The Journal of the Acoustical Society of America, vol. 51, no. 5B, pp. 1606-1608, 1972.

[14] L. Brillouin, "Wave propagation in periodic structures: electric filters and crystal lattices," Courier Corporation, 2003.

[15] D. S. Drumheller, "Acoustical properties of drill strings," The Journal of the Acoustical Society of America, vol. 85, no. 3, pp. 1048-1064, 1989.

[16] J. M. Carcione and F. Poletto, "Simulation of stress waves in attenuating drill strings, including piezoelectric sources and sensors," The Journal of the Acoustical Society of America, vol. 108, no. 1, pp. 53-64, 2000.

[17] W. C. Chin, "Wave Propagation in Drilling, Well Logging and Reservoir Applications," John Wiley \& Sons, 2014.

[18] T. Wiens, "Kasami sequences, m-sequences, linear feedback shift registers," https://www.mathworks.com/matlabcentral/fileexchange/22716.

[19] P. Koopman, "Maximal length lfsr feedback terms," n.d. https://users.ece.cmu.edu/ koopman/lfsr/index.html.

[20] J. ven der Buhs, "Investigation and Optimization of Hydraulic Step-down Switched Inertance Converters with Non-uniform Inertance Tubes", M.Sc. thesis, Department of Mechanical Engineering, University of Saskatchewan, Saskatoon, Canada, 2017.

[21] C. H. Sherman, J. L. Butler, "Transducers and Arrays for Underwater Sound," Springer, New York, 2007.

[22] P. Krus, K. Weddfelt and J. O. Palmberg, "Fast Pipeline Models for Simulation of Hydraulic Systems," Journal of Dynamic Systems Measurement and Control, 1994.

[23] C. Bayliss, "Application and Development of Finite Element Techniques for Transducer Design and Analysis," PhD Thesis, The University of Birmingham, Birmingham, UK, 1998.

[24] D. A. Berlincourt, D. R. Curran and H. Jaffe, "Piezoelectric and piezomagnetic materials and their function in transducers," Physical Acoustics, Vol. 1, Part A, ed. by W.P. Mason, Academic Press, New York, 1964.

[25] N. Johnston, "The Transmission Line Method for Modelling laminar flow of Liquids in Pipelines," Journal of Systems and Control Engineering, pp. 586-597, 2012.

[26] N. Johnston, M. Pan and S. Kudzma, "An Enhanced Transmission Line Method for Modelling Laminar Flow of Liquid in Pipelines," Journal of Systems and Control Engineering, vol. 228, no. 4, pp. 193-206, 2013.

[27] J. ven der Buhs, T. Wiens, "Modelling Dynamic Response of Hydraulic Fluid Within Tapered Transmission Lines," Proceedings of the 15th Scandinavian International Conference on Fluid Power, Linköping, Sweden, 2017. 